\title{
An mHealth Solution based on Social Cognitive Theory to Promote Adherence to Cardiac Rehabilitation for Cardiac Patient
}

\author{
Zhiqiang Luo, Durairaj Ponraj, Nam Beng Tan, Swee Yaw Tan, Jack Wei Chieh Tan
}

\begin{abstract}
The present project aims to develop a mHealth solution leveraging on network and mobile technologies to assist patients with cardiovascular disease to promote their adherence to cardiac rehabilitation exercise. The software of solution comprises two mobile apps on smart phones and one website on Windows Azure cloud, where two apps communicates with website via mobile services. The design of software is based on the proven behavior change strategy to improve patient's self-efficacy, self-regulation, and social support in cardiac rehabilitation. First, to improve selfefficacy, the solution can continuously summarize patient's achievements, illustrate role model and provide therapeutic suggestions and motivation messages. Second, to promote selfregulation, the solution can bring the therapeutic plan to patient's mobile phone, set appropriate short- and long-term goals and real-time monitor the performance using heart rate monitor. Last, the solution can help patients to share their progress with friends and family members via group chatting, sharing, and messaging on smart phones. The system is currently tested by a randomized controlled pilot study at the rehabilitation centre with National Heart Centre Singapore.
\end{abstract}

Keywords-mHealth, mobile cloud, mobile computing, social computing

\section{Introduction}

While it is well established that cardiac rehabilitation (CR) exercise therapy can lead to reliable changes in both physiological and functional outcomes for patients with cardiovascular disease (CVD), the adherence to these (exercise) programs is problematic (American Association of Cardiovascular and Pulmonary Rehabilitation (AACVPR) [1]). Two factors are identified to explain problems of the adherence and maintenance during and after CR: a) the sufficient assistance to $\mathrm{CR}$ and behavior change, and b) the participant motivation that is inferred from behavioral manifestations of adherence like effort and persistence. Thus American Heart Association (AHA) Presidential Advisory called for a reengineering of CR to enhance access, adherence, and effectiveness [2].

\section{A. mHealth for cardiac rehabilitation}

mHealth provides the opportunity to improve access to health promotion interventions and has the unique advantage of being able to influence health behaviours in real-time [3]. mHealth programs, based on mobile phones and Internet technology, can be delivered anywhere at any time. Communication between therapists or nurses with patients can be regular and frequent, and messages can be delivered in a time-sensitive or time-appropriate way to support behaviour change in more continuous manner. One early trial of mHealth is to deliver CR content via short-message service (SMS) [4].
Recently, the mobile apps-based mHealth programs, especially those promoting physical activity participation and adherence, become popular. Under the Health and Fitness category on Apple Store, there are more than 90 apps to assist workout and exercise; in Google Play, there are more than 200 apps under search keyword "cardiac monitor \& fitness". Mobile apps can greatly impact physical activity because of two unique advantages: (a) real-time monitoring physical activity performances and (b) interfacing with accelerometers, pedometers, and other wireless devices that track physical activity and biometric data. Mobile apps can support users to measure exercise capacity too. For example, the GPS sensor with mobile phone can help patients to conduct 6-minute walk test to measure the walking distance. The measurement of exercise capacity can be integrated with peripheral sensors for the measurements of ECG, heart rate, blood pressure, weight and more.

\section{B. Self-efficacy and self-regulation}

Self-efficacy and self-regulation are constructs of social cognitive theory (SCT), one widely recognized and proven theory for behaviour change [5]. Self-efficacy is commonly defined as the belief in one's capabilities to achieve a goal or an outcome, for example, exercising control over one's health habits. Self-efficacy is closely related to the exercise adherence behaviour. Once patients find that the goal can be achieved within their capability, they most probably perform specific actions, even in the face of adversity. The degree of self-efficacy has been consistently identified as determinants of exercise adherence, especially in asymptomatic, younger, and older populations [6]. Bandura [7] pointed out that there are four sources for self-efficacy: mastery exposures (e.g. performance accomplishments), vicarious experience (e.g. role modelling), verbal persuasion, and emotional arousal. These four sources can be delivered via voice, text, resident application, mobile web, or other modality on mobile phone, which can be accessed in real-time, more frequently and interactively than any previous means like the internet- or manual-based intervention.

Self-regulation refers to efforts by individual to alter his/her thoughts, feelings, desires, and actions in the service of attaining distal goals, for example, self-monitoring of health-related behaviour. Maes \& Karoly [8] conceptualized self-regulation via a goal-guidance model to emphasize the implementation and maintenance strategies to achieve behavioural changes. People have to learn to monitor their health behaviour and the circumstance under which it occurs, and how to use proximal goals to motivate them and guide their behaviour. The physiological monitoring based on mobile technology can assist patients to real-time and continually monitor health status and provide just-in-time intervention in the circumstance of rehabilitation [9]. People 
also need to learn how to enlist social supports to sustain their efforts [10]. The current fitness apps can share user's physical activity with friends via website and even social media to motivate user's participation and adoption of mobile app.

\section{Objective of this study}

There are two gaps in previous studies about employing $\mathrm{mHealth}$ for CR. First, as study [10] found, there are only a few mHealth interventions targeting at $\mathrm{CR}$ that are based on validated psychological theories. Because of this limitation, the existing mHealth programs yielded limited outcomes for $\mathrm{CR}$ adherence to prove the efficacy and advantage. It calls for more research and practices to design contents of mHealth for CR based upon the psychological theories.

Second, the main attention of previous studies has been paid to enhance self-efficacy to adopt exercise program. Few studies provide details on how to enhance strategies to effectively and efficiently do exercise and sustain participant's efforts on exercise, especially the social supports gained from family or friends.

The objective of this study is to develop an mHealth solution, namely cardiac rehabilitation mate (simply CRMate), leveraging on the social cognitive theory to improve patient's self-efficacy, self-regulation, and social support in cardiac rehabilitation. It is expected that, through using the solution, cardiac patients can better adhere to cardiac rehabilitation exercise.

\section{System Overview}

The proposed mHealth solution, CRMate, comprises three software: one website and two apps on smart phone (one app for patient and one app for patient's family members), as shown in Figure 1. The website is hosted in Windows Azure cloud platform and serves therapist to operate. The CRMate website for therapist is to support therapist to design and update therapy plan for patient, monitor patient's progress, manage patient's registration, and communicate with patient via sending SMS through one third-party SMS engine. The patient's rehabilitation progress, therapy plan details, and communication messages will be stored in Azure Storage. The profiles of both patient and therapist, meta-information of group and therapy plan, and family contacts will be stored in database. The communication between website and two apps is done by mobile services.

The CRMate app for patient is to support patient's rehabilitation. It starts from receiving therapy plan on website via mobile service and save therapy plan into local database. The app has three major features to promote selfregulation, self-efficacy, and social support, respectively, which will be introduced in details in Section 3. The app can connect heart rate monitoring sensor via Bluetooth connection to real-time record patient's heart rate during exercise. After each exercise session, the exercise result will saved into the local database as well as sent to online database at website for therapist's review.

Meanwhile, the app can send notification to peer patients via notification to update them the new exercise result. The notification is only received by the same app installed at patient's smart phone. Besides the peer patients, the app can send the exercise result to patient's family via SMS. The family member can also get the exercise information from the storage of website in the app for family.

The CRMate app for family is to help family member to know patient's therapy plan, update patient's progress and accomplishment in rehabilitation.

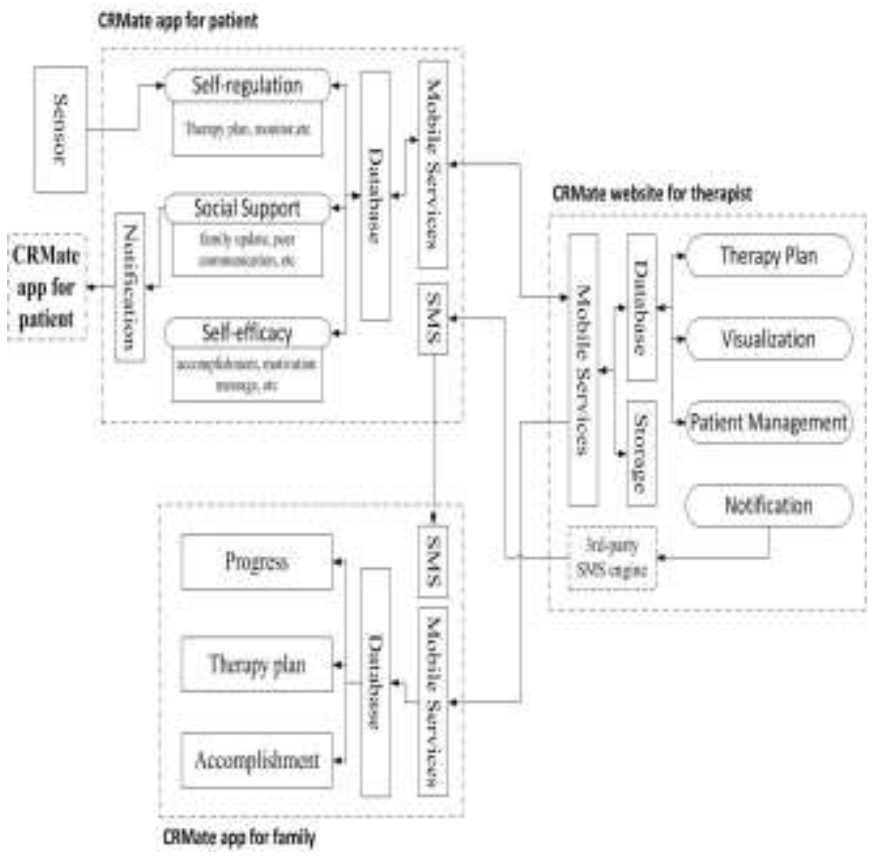

Figure 1. The CRMate system architecture.

\section{System Features}

There are three major features of CRMate to promote patient's self-efficacy, self-regulation, and social support, respectively. The CRMate system embodies the findings and rules of social cognitive theory, which are proven to support the behavior change and adherence.

\section{A. Features to promote self-efficacy}

The accomplishment of exercise tasks will be determined during cardiac rehabilitation. The data to determine accomplishment includes the maximum and average heart rates (HR) when taking exercise task, the total calories burned, and the total duration of exercise task. There are three levels of accomplishment with CRMate: Gold, Silver, and Bronze. The role model is to show the previous patient who successfully recovered through the current cardiac rehabilitation practice. Therapist will compare patient's current performance against the role model and give an evaluation, e.g. Good, OK, or Not Bad. The therapeutic suggestion and motivation messages from therapists could motivate patient to adopt physical activity. A private and instant communication channel is built to allow therapist to send SMS directly to the target patient via the website of the developed system. The target patient can reply therapist via mobile app. 


\section{B. Features to promote self- regulation}

The monitoring of physical activity is implemented by measuring the performance of exercise in real time, such as heart rate, calories, etc. The mobile app can connect to a heart rate monitor device to measure heart rate in real time. The mobile app can automatically calculate the burned calories, the distance travelled, and the duration of exercise. The data collected in each exercise session will be wirelessly sent to online Azure database and storage for therapist to check in website. The data will be shown in rich graphics, like line chart and bar chart. Thus therapist can have a completed view on the whole performance. The screenshots of monitoring functions implemented in CRMate website and app are shown in Figure 2.
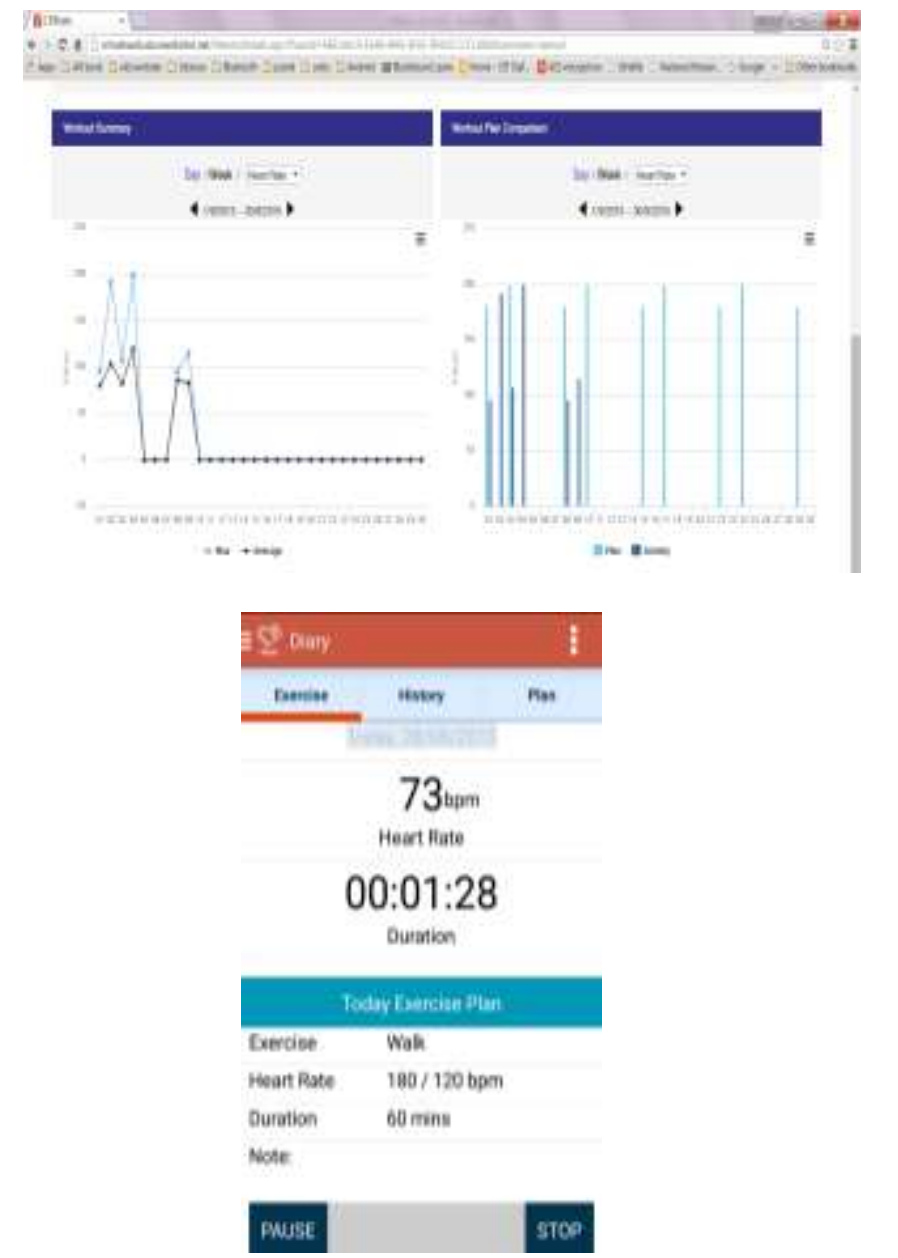

Figure 2: the two screenshots of monitoring patient's performance in exercise: The monitoring on website (up) to see the monthly performance and the monitoring on mobile app (down) to check out heart rate and duration in real time

The guide of therapy plan is a dynamic process. It starts from the very beginning of rehabilitation when a doctor or therapist defines the therapy goal and plan on the CRMate website. More particularly, the therapy plan defines a series of exercise tasks at the required dates in three-month cardiac rehabilitation. The exercise tasks include the exercise type, the goals of each task, the duration of exercise, etc. The therapy plan can be retrieved by patient's mobile app. At the date of doing exercise, the CRMate app can remind patient and display today's exercise plan in app for guide.

\section{Features to promote social support}

The CRMate also incorporate the social network function. The peer support from patients of the same CR group is done via the communication within CRMate apps. The communication is similar as the popular chatting app, e.g. Whatapp. They can send text to each other to communicate. Meanwhile, patients exercise status can be automatically updated in the same group. The family support is implemented by sharing patient's exercise records with family members. In particular, the family member can use the CRMate app for family to retrieve whole patient's therapy plan and exercise progress. The family support is crucial for patient to adhere to exercises

\section{Iv. Pilot Test}

The CRMate solution is now being tested by a clinic trial with National Heart Centre Singapore. Before the clinic trial, four physiotherapists with this centre have the first use of this system. They answer a questionnaire to evaluate the features on helping their patients in cardiac rehabilitation and the usability of CRMate system. All therapists acknowledged that the system could play positive roles to support their patients to do exercise effectively. They are particularly keen to use the social network features, like helping patients to form interest groups and real-time updating exercise records with patient's family. They feel fine with the interface of both app and website in terms of ease of use and understanding. Some comments are given to the navigation in app and the fast internet connection. One reason maybe that some patients' smart phones don't not have the internet connection, or the connection may not be good in some places within a building.

\section{v. Conclusion}

A new mhealth solution CRMate, leveraging on network and mobile technologies to assist cardiac patients to adhere to exercise in cardiac rehabilitation. The development of CRMate is based on social cognitive theory to effectively support patient's behavior change in exercise. The main difference between CRMate and other mHealth solutions for cardiac rehabilitation is that CRMate builds one social network feature to support the real-time communications among patients, therapist, and patient's family. The feature is implemented via using the notification technique with current mobile technique and mobile services with cloud technology to facilitate multiple mobile devices connection. The mHealth solution in mobile cloud could bring the closer interactions among these stakeholders in cardiac rehabilitation. CRMate can play a complementary role to promote the effectiveness and adherence of the exercisebased cardiac rehabilitation conducted in both hospital and community settings. 


\section{Acknowledgements}

The project is supported by Singapore Heart Foundation with project No.RG2013/04

\section{References}

[1] American Association of Cardiovascular and Pulmonary Rehabilitation, "Guidelines for cardiac rehabilitation and secondary prevention programs," 3rd edition. Champaign, IL: Human Kinetics; 1999.

[2] G.J. Balady, P.A. Ades, V.A. Bittner, B.A. Franklin, N.F. Gordon, et al. "American Heart Association Science A, Coordinating C. Referral, enrolment, and delivery of cardiac rehabilitation/secondary prevention programs at clinical centers and beyond: a presidential advisory from the American Heart Association," Circulation 124:2951-2960, 2011.

[3] W.T. Riley, D.E. Rivera, A.A. Atienza, W. Nilsen, S.M. Allison, R. Mermelstein, "Health behaviour models in the age of mobile interventions: are our theories up to the task?" Translational Behaviour Medicine, 1(1):53-71, 2011;

[4] B. Fjeldsoe, A. Marshall, and Y. Miller, "Behaviour change interventions delivered by mobile telephone short-message service," American Journal of Preventive Medicine, 36:165-173, 2009.

[5] A. Bandura, "The primacy of self-regulation in health promotion," Applied Psychology: An International Review, 54(2): 245-254, 2005.

[6] E. McAuley, M.M. Pena, and G. Jerome, "Self-efficacy as a determinant and an outcome of exercise," In G. C. Roberts (Ed.), Advances in motivation in sport and exercise. Champaign, IL: Human Kinetics Publishers, p. 235-261, 2011.

[7] A. Bandura, "Self-efficacy: Toward a unifying theory of behavioural change," Psychological Review, 84(2): 191-215, 1977.

[8] S. Maes and P. Karoly, "Self-regulation assessment and intervention in physical health and illness: A review," Applied Psychology: An International Review, 54(2), 245 - 277, 2005.

[9] C. Bexelius, M. Lof, S. Sandin, et al. "Measure of physical activity using cell phones: Validation using criterion measures," Journal of Medical Internet Research, 12(1):e2, 2010.

[10] A. Bandura, "Health promotion by social cognitive means," Health Education \& Behaviour, 31(2):143-64, 2004.

[11] A.L. Beatty, Y. Fukuoka, M. Whooley, "Using mobile technology for cardiac rehabilitation: A review and framework for development and evaluation," Journal of the American Heart Association, 2(6): 1-8, 2013.

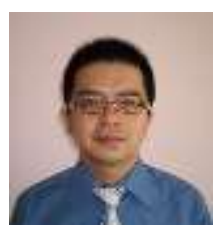

Dr Zhiqiang Luo is a lecturer with School of Information Technology, Nanyang Polytechnic, Singapore. His research interests include mobile computing, mHealth, cloud computing, an human computer interaction. Dr Luo is a member of ACM and IEEE. 